\title{
Leptin enhances glycolysis via OPA1-mediated mitochondrial fusion to promote mesenchymal stem cell survival
}

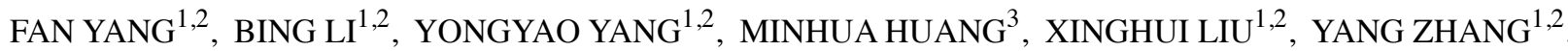

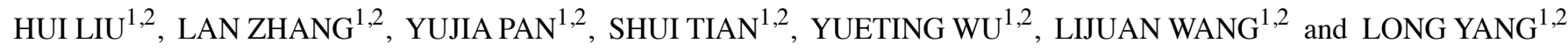 \\ ${ }^{1}$ Department of Cardiology, Guizhou Provincial People's Hospital, Guiyang, Guizhou 550002; \\ ${ }^{2}$ Research Institute of Cardiovascular Disease, Guiyang, Guizhou 550001; \\ ${ }^{3}$ Xixiu District People's Hospital, Anshun, Guizhou 561000, P.R. China
}

Received December 7, 2018; Accepted April 24, 2019

DOI: 10.3892/ijmm.2019.4189

\begin{abstract}
Transplantation of mesenchymal stem cells (MSCs) is emerging as a potential therapy for cardiovascular diseases. However, the poor survival of transplanted MSCs is a major obstacle to improving their clinical efficacy. Accumulating evidence indicates that hypoxic preconditioning (HPC) can improve the survival of MSCs. It has been previously reported that leptin plays a critical role in HPC-enhanced MSC survival through increasing optic atrophy 1 (OPA1)-dependent mitochondrial fusion. Survival of MSCs mainly relies on glycolysis as an energy source. The close relationship between leptin and glucose homeostasis has attracted intense scientific interest. Furthermore, emerging evidence indicates that mitochondrial dynamics (fusion and fission) are associated with alterations in glycolysis. The aim of the present study was to investigate whether leptin increases MSC survival through metabolic regulation. Leptin-modulated increased OPA1 expression was found to be associated with increased glycolysis. However, the glycolytic efficacy of leptin was abrogated after silencing OPA1 using a selective siRNA, suggesting that OPA1 directly regulates glycolysis. Furthermore, the activation of sodium-glucose symporter 1 (SGLT1) was markedly induced by leptin. However, leptin-induced glycolysis was primarily
\end{abstract}

Correspondence to: Dr Long Yang, Department of Cardiology, Guizhou Provincial People's Hospital, 83 Zhongshan East Road, Guiyang, Guizhou 550002, P.R. China

E-mail: yanglong1001@163.com

Abbreviations: HPC, hypoxic preconditioning; OPA1, optic atrophy 1; VDAC, voltage-dependent anion channel; TEM, transmission electron microscopy; OXPHOS, oxidative phosphorylation; OCR, oxygen consumption rate; GAPDH, glyceraldehyde-3-phosphate dehydrogenase; HK-1, hexokinase-1; LDH- $\alpha$, lactate dehydrogenase- $\alpha$; PDH- $\alpha$, pyruvate dehydrogenase- $\alpha$; cpt- $1 \alpha$, carnitine palmitoyltransferase- $1 \alpha$; GLUTs, glucose transporters; SGLT1, sodium-glucose symporter 1; EF, ejection fraction

Key words: leptin, mesenchymal stem cells, glycolysis, optic atrophy 1 , sodium-glucose symporter 1 blocked by SGLT1 inhibitor treatment. Thus, leptin regulates OPA1-dependent glycolysis to improve MSC survival primarily through SGLT1 activation. We therefore identified a pivotal leptin/OPA1/SGLT1 signaling pathway for mitochondrial dynamic-mediated glycolysis, which may optimize the therapeutic efficiency of MSCs.

\section{Introduction}

Mesenchymal stem cell (MSC)-based therapies for obesity, acute myocardial infarction (MI) and graft-versus-host disease (GvHD) have been proven to be highly efficient (1-3), emerging as promising approaches to regenerative medicine. Extensive evidence suggests that transplantation of MSCs is characterized by hypoimmunogenicity (4) and multipotent differentiation potential, and is in accordance with ethical guidelines. However, the poor retention and survival of the transplanted MSCs limit their therapeutic efficacy in clinical trials. Previous studies have verified that hypoxic preconditioning (HPC) promotes the survival of MSCs after transplantation $(5,6)$. Furthermore, leptin has been confirmed to play a vital role in HPC-elicited resistance of MSCs to apoptosis (7).

Energy production by glycolysis is crucial for cell fate, particularly in the absence of oxygen. The interesting aspect of MSCs is that they preferentially use glycolysis in an undifferentiated or anti-apoptotic state, whereas they rely on mitochondrial oxidative phosphorylation (OXPHOS) during their proliferative phase (6). Leptin, a peptidase secreted by adipocytes, is closely associated with the peripheral signaling pathway of glucose homeostasis (8). A recent study demonstrated that leptin increased mitochondrial fusion through the glycogen synthase kinase (GSK)3/OMA1/optic atrophy (OPA)1 signaling pathway to prevent apoptosis of MSCs in a hostile microenvironment (9).

Mitochondria are highly dynamic organelles that can move and change their morphology through fission and fusion. Sustainable dynamic homeostasis of fusion and fission is responsible for maintaining mitochondrial respiration, morphology and content. OPA1 is a mitochondrial inner membrane protein that has at least five isoforms, which mediate fusion and fission in the inner mitochondrial 
membrane; the long OPA1 isoforms (L-OPA1) are associated with fusion, whereas the short OPA1 isoforms (S-OPA1) are associated with fission (10-12). Emerging evidence indicates the potential of regulating mitochondrial dynamics in metabolic alterations (13). In hypothalamic pro-opiomelanocortin neurons, mitochondrial fragmentation suppresses the response to glucose in a fed state (14). In activated effector $\mathrm{T}$ cells, mitochondrial fusion increases glycolysis to promote cellular immunotherapy against cancer cells (15). However, there is no research directly indicating whether OPA1-induced stimulation of glycolysis is involved in the rescue of MSCs following leptin treatment. The aim of the present study was to determine whether leptin targeting OPA1 increases glycolysis in MSCs to improve their survival rate in response to hypoxia, and to investigate the underlying mechanism.

\section{Materials and methods}

Cell isolation, culture and hypoxic injury. Human bone marrow was collected from patients undergoing hip replacement surgery after obtaining their informed consent. The study protocol was approved by the Human Ethics Committee of Guizhou Provincial People's Hospital (Guiyang, China). MSCs were isolated and cultured as previously reported (9).

MSCs were cultured with human recombinant leptin (50 ng/ml; R\&D Systems, Inc.) in Dulbecco's modified Eagle's medium (DMEM; Corning, Inc.) with $10 \%$ fetal bovine serum (FBS; Thermo Fisher Scientific, Inc.) under normoxic conditions for $24 \mathrm{~h}$, and were then exposed to DMEM without glucose or FBS in a hypoxic chamber $\left(0.5 \% \quad \mathrm{O}_{2} / 5 \% \mathrm{CO}_{2}\right.$; BioSpherix, Ltd.) for an additional $24 \mathrm{~h}$.

Cell siRNA transfection. A siRNA silencing the expression of human OPA1 (siOPA1) and a scramble siRNA (NC) were purchased from GenePharma. siRNA transfection was coordinated with Lipofectamine RNAi MAX (Invitrogen; Thermo Fisher Scientific, Inc.) according to the manufacturer's instructions. Transfection of $50 \mathrm{nM}$ siRNA and the specific sequences of OPA1 (sense, CCAUGUGGCCCUAUUUAA ATT; antisense, UUUAAAUAGGGCCACAUGGTT) were conducted as described previously (9).

Annexin V/PI staining. The harvested cells $\left(2 \times 10^{5}\right.$ cells per sample) were incubated with a mixture of Annexin $\mathrm{V}$ and PI for $30 \mathrm{~min}$ according to the manufacturer's instructions (Dojindo Molecular Technologies, Inc.). Apoptotic cells were quantified by flow cytometry (BD Biosciences).

Oxygen consumption rate (OCR). The oxygen consumption of the intact cells was measured as described previously $(16,17)$. Briefly, the harvested cells $\left(1 \times 10^{6}\right.$ cells $)$ were resuspended with $1 \mathrm{ml}$ MiR05 [containing $0.5 \mathrm{mM}$ EGTA, $3 \mathrm{mM}$ $\mathrm{MgCl}_{2} \cdot 6 \mathrm{H}_{2} \mathrm{O}, 60 \mathrm{mM}$ potassium lactobionate, $20 \mathrm{mM}$ taurine, $10 \mathrm{mM} \mathrm{KH}_{2} \mathrm{PO}_{4}, 20 \mathrm{mM}$ HEPES, $110 \mathrm{mM}$ sucrose and $1 \mathrm{~g} / \mathrm{l}$ fatty acid-free bovine serum albumin (BSA) at $\mathrm{pH}$ 7.1], and were then transferred into the experimental chamber. Subsequently, using Oroboros Oxygraph-2k, the OCR was analyzed by titrating various substrates/inhibitors of the respiratory chain in a stepwise manner at $30^{\circ} \mathrm{C}$ (Oroboros Instruments $\mathrm{GmbH}$ ).
Extracellular acidification rate (ECAR). ECAR was measured by FLUOstar Omega (BMG LABTECH GmbH) according to the manufacturer's instructions. Briefly, MSCs were plated in a 96-well plate $\left(1 \times 10^{4}\right.$ cells/well) overnight. The next day, after replacing with fresh culture media, $10 \mu \mathrm{l}$ reconstituted $\mathrm{pH}-\mathrm{Xtra}$ reagent was added into each well (a well with $10 \mu \mathrm{l}$ fresh culture media without cells was used as blank control). Subsequently, $100 \mu \mathrm{l}$ mineral oil was promptly added into each well, and fluorescence intensity was measured by a fluorescence plate reader (380 $\mathrm{nm}$ excitation and $615 \mathrm{~nm}$ emission).

Western blot analysis. Western blotting was conducted as described previously (7). Proteins were extracted from MSCs using RIPA solution (Beyotime Institute of Biotechnology) with protease inhibitor cocktail (Roche Diagnostics). Protein concentrations were quantified using BCA Protein Assay Kit (Thermo Fisher Scientific, Inc.) and separated by sodium dodecyl sulfate-polyacrylamide gel electrophoresis (8-10\%), followed by transfer to polyvinylidene difluoride membranes (EMD Millipore). After blocking with 5\% BSA for $1 \mathrm{~h}$ at room temperature, the proteins of interest were incubated with primary antibodies overnight at $4^{\circ} \mathrm{C}$ : Cleaved caspase-3 (1:500, Cell Signaling Technology, Inc.); voltage-dependent anion channel (VDAC) (1:500, Santa Cruz Biotechnology, Inc.); poly-OPA1 (1:1,000, BD Biosciences); glucose transporter (GLUT)4 (1:500, Santa Cruz Biotechnology, Inc.); GLUT1 (1:1,000, Abcam); sodium-glucose symporter (SGLT)1 (1:1,000, Abcam); $\beta$-actin (1:3,000, KangChen Biotech. Co., Ltd.). On the following day, the membranes were incubated with anti-rabbit and anti-mouse secondary antibody (1:5,000, Abcam) for $1 \mathrm{~h}$ at room temperature, and then detected using the Gel Doc EZ Imaging System (Bio-Rad Laboratories, Inc.) with an ECL kit (Merck KGaA), and analyzed using Image Lab software (Bio-Rad Laboratories, Inc.).

Reverse transcription-quantitative polymerase chain reaction $(R T-q P C R)$ analysis. The gene expression levels were measured by extracting total RNA using TRIzol reagent (Invitrogen; Thermo Fisher Scientific, Inc.), according to the manufacturer's instructions. RT-qPCR was performed as previously described (18). Briefly, the RNA concentration was measured by a NanoDrop spectrophotometer (Thermo Fisher Scientific, Inc.). Total RNA ( $2 \mu \mathrm{g})$ was reverse-transcribed to cDNA using a Reverse Transcription kit (Takara). RT-qPCR was performed using SYBR Green PCR Master Mix (Takara). The cycling conditions were as follows: $95^{\circ} \mathrm{C}$ for $10 \mathrm{~min}$, followed by 40 cycles of $15 \mathrm{sec}$ at $95^{\circ} \mathrm{C}$ and $30 \mathrm{sec}$ at $60^{\circ} \mathrm{C}$. mRNA levels were normalized to $\beta$-actin and determined using the $2^{-\Delta \Delta \mathrm{Cq}}$ method. The gene primers are listed in Table I.

Transmission electron microscopy (TEM). Mitochondrial ultrastructure was examined by TEM. Briefly, the cells were fixed with $2.5 \%$ glutaraldehyde for at least $4 \mathrm{~h}$. After washing with phosphate-buffered saline, the cells were post-fixed with $1 \% \mathrm{OsO}_{4}$ for $1-2 \mathrm{~h}$. Then, following dehydration by the ethanol gradient method, the cells were infiltrated by acetone overnight. Subsequently, the specimens were embedded in Spurr resin, heated at $70^{\circ} \mathrm{C}$ for $9 \mathrm{~h}$ and sectioned with Leica EM UC7 Ultramicrotome (Leica Microsystems $\mathrm{GmbH}$ ). Uranyl acetate and alkaline lead citrate were used to stain the sections. 
Table I. Human primers for quantitative polymerase chain reaction analysis.

Primer sequences 5'-3'

\begin{tabular}{lll}
\cline { 2 - 3 } Gene & \multicolumn{1}{c}{ Forward } & \multicolumn{1}{c}{ Reverse } \\
\hline GAPDH & \multicolumn{1}{c}{ TCAACGTGTGGTCATCTCCG } \\
H 1 & ATGGTGGATGGTGAGCAAGG & TGGTCATCAACCCTTCCACG \\
$P D H-\alpha$ & GTTCGGAGGACCCAGCAATTA & CAGGAACCCTCGTTTGGTGA \\
$c p t-1 \alpha$ & GAGTTTGTGTGGGTTCTCCA & GGCTCCTACAGCAAGGACAC \\
GLUT1 & ATGACGGCTATGGTGTGTCG & GTGGGTCGCTGGAGTAGATG \\
GLUT3 & ACAACCAGACATGGGTCCAC & TTCCAGCCCAGCACATGAAC \\
GLUT5 & CGAGACCCAGAGATGCTGTAA & AGGACTTGCCCAGTTCGAG \\
GLUT9 & GGCTCTACCCCGATGGTTTC & CTGTGTCCCCATCGCTGTAAT \\
SGLT1 & GACCAGGTTCCTGAAAGGGT & GTGTCCCTGGTGAAGAGTGC \\
actin & GCACCAGGCACTGGAATTAAAC & AGATACACCCCTTCCTTCATGC \\
\hline
\end{tabular}

HK, hexokinase; LDH, lactate dehydrogenase; PDH, pyruvate dehydrogenase; cpt, carnitine palmitoyltransferase; GLUT, glucose transporter; SGLT1, sodium-glucose symporter 1 .

Images were captured randomly using Hitachi H-7650 TEM (Hitachi, Ltd.).

Periodic acid-Schiff (PAS) staining and glucose oxidase method. Glycogen content was measured by PAS staining according to the manufacturer's instructions (SenBeiJia Biotechnology, Co.). Briefly, the harvested cells were fixed by PAS fixing liquid for $10 \mathrm{~min}$. After washing and drying, the cells were incubated in periodic acid solution at room temperature for $15 \mathrm{~min}$ and stained by Schiff reagent for $30 \mathrm{~min}$ in the dark. The images were captured randomly at a magnification of $\mathrm{x} 200$. Glucose content was detected using the glucose oxidase method according to the manufacturer's instructions (Applygen Technologies, Inc), and absorbance was measured at $570 \mathrm{~nm}$ by a microplate reader (Bio-Rad Laboratories, Inc.).

ATP measurement. Cellular ATP content was analyzed by a luciferin/luciferase-based kit according to the manufacturer's instructions (Beyotime Institute of Biotechnology).

Lactate measurement. The lactate content was measured using a lactate assay kit (Jiancheng Biotechnology) according to the manufacturer's instructions.

Sotagliflozin (LX4211) treatment assay. MSCs were incubated with leptin $(50 \mathrm{ng} / \mathrm{ml})$ under normal culture conditions for $24 \mathrm{~h}$. Subsequently, these MSCs were cultured with leptin in the presence or absence of $36 \mathrm{nM}$ sotagliflozin (LX4211; Selleck Chemicals), an inhibitor of SGLT1, and subjected to hypoxia and glucose + serum deprivation for an additional $24 \mathrm{~h}$.

MI mouse model and MSC transplantation. A MI mouse model was induced by ligation of the left anterior descending coronary artery in 10-12-week-old male C57BL/6J mice (20-25 g), as previously described (7). MSCs $\left(1.5 \times 10^{5}\right.$ cells in
$20 \mu \mathrm{l}$ DMEM per mouse) were transplanted to the border zone of the infarct.

Sirius Red staining. The mice were euthanized, and the hearts were harvested and embedded in paraffin on day 28 post-MI. The tissue samples were cut into $3-\mu \mathrm{m}$ sections and stained with Sirius Red (Beijing Solarbio Science \& Technology Co., Ltd.), as described previously (19). Infarct areas were analyzed according to the sum of the endocardial and epicardial length of the infarct zone in proportion to the total length of the endocardial and epicardial left ventricle using Image-Pro-Plus software (Media Cybernetics, Inc.).

Echocardiography. Echocardiography was performed on day 28 post-MI, as described previously (20). Cardiac morphology and function were analyzed via a Vevo 2100 system (VisualSonics, Inc.). Left ventricular ejection fraction was measured for at least three cardiac cycle intervals.

Statistical analysis. All experiments were performed at least three times. All data are presented as mean \pm standard error of the mean. Student's t-test was used to determine significance when comparing two sets of data. One-way analysis of variance followed by Tukey's post hoc test was used for comparison of more than three sets of data. All data were analyzed using SPSS 17.0 (SPSS Inc.). The statistics were presented by GraphPad Prism 5 (GraphPad Software, Inc.). P $<0.05$ was considered to indicate statistically significant differences.

\section{Results}

Leptin enhances the survival of MSCs by preventing apoptosis in vitro. In order to examine the protective effect of leptin on MSCs in response to the microenvironment after transplantation, MSCs were incubated with recombinant leptin (MSCs-Lep) for $24 \mathrm{~h}$ under normal culture conditions, and 
A

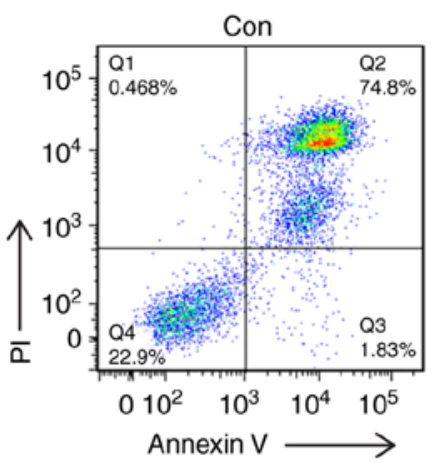

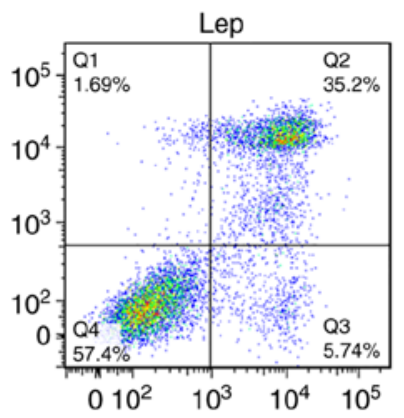

B
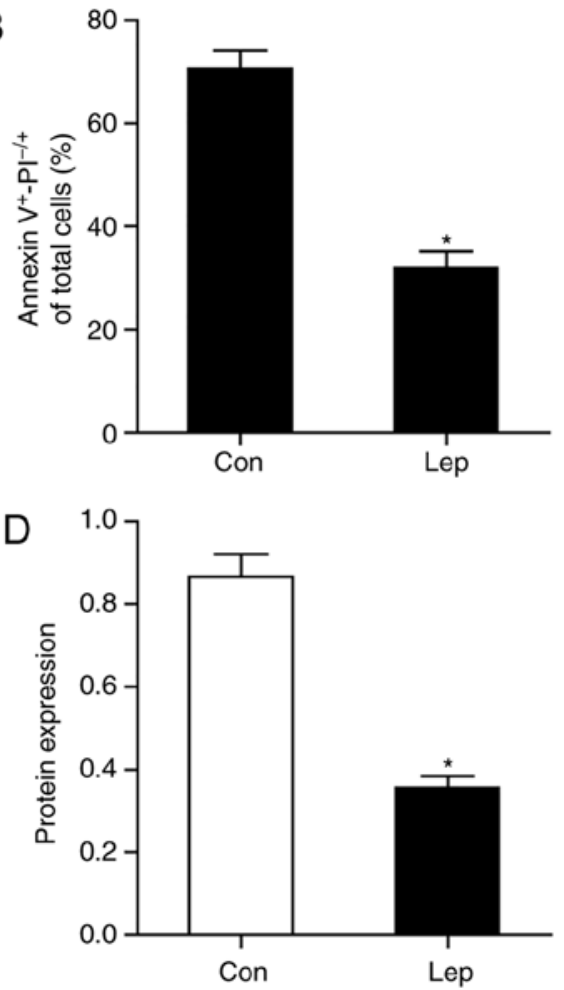

Figure 1. Leptin endowed MSCs with resistance to apoptosis under hypoxic conditions. (A) Apoptotic MSCs were distinguished by Annexin- $\mathrm{V}^{+}$and $\mathrm{PI}^{-} /^{+}$ staining and analyzed by FACS during hypoxia $\left(0.5 \% \mathrm{O}_{2}\right)$ and glucose + serum deprivation for $24 \mathrm{~h}$. (B) Early (Q3) and late (Q2) apoptotic cells were simultaneously calculated. (C) Cleaved caspase 3 (cle caspase 3) protein expression in whole-cell lysates was detected by western blotting. Protein content was normalized using $\beta$-actin as control. (D) Densitometry was used to quantify protein expression levels. Each in vitro experiment was repeated three times. Data are presented as mean \pm standard error of the mean; $\mathrm{n}=3\left({ }^{*} \mathrm{P}<0.05\right)$. MSCs, mesenchymal stem cells; PI, propidium iodide.

then exposed to hypoxia and glucose + serum deprivation for an additional $24 \mathrm{~h}$ in vitro. The solvent of leptin, Tris buffer, was used as control (MSCs-Con).

To distinguish the apoptotic cells affected by hypoxia, the percentage of Annexin $\mathrm{V}^{+}$and $\mathrm{PI}^{-/+}$staining was calculated by flow cytometry. Decreased apoptotic ratio was detected in MSCs-Lep, while a higher percentage of Annexin $\mathrm{V}^{+}$and $\mathrm{PI}^{-/+}$staining was detected in MSCs-Con (Fig. 1A and B). Similarly, lower expression of cleaved caspase-3 was observed in MSCs-Lep compared with MSCs-Con (Fig. 1C and D). These data suggest that leptin exerted a cytoprotective effect on MSCs against hypoxic insults.

Increased mitochondrial fusion is induced by leptin. Mitochondrial morphology is closely associated with cell fate; an abundance of pro-apoptotic factors is stored in mitochondrial cristae, and these pro-apoptotic proteins are released from the mitochondria into the cytoplasm to activate the apoptosis signaling pathway (21-23). First, mitochondrial morphology was evaluated by TEM. Elongated tubular mitochondria were prominent in MSCs-Lep under hypoxic stress, while the mitochondria observed in MSCs-Con were fewer and sparsely distributed (Fig. 2A). In contrast to MSCs-Con, increased mitochondrial length and area were observed in MSCs-Lep (Fig. 2B). Moreover, the increased protein level of VDAC indicated that the mitochondrial content was higher in MSCs-Lep compared with MSCs-Con (Fig. 2C and D).

The different isoforms of OPA1 modulate mitochondrial inner membrane dynamics, among which L-OPA1 (isoforms a-b, 85-100 $\mathrm{kDa}$ ) are implicated in fusion, whereas S-OPA1 (isoforms c-e, $\sim 75-85 \mathrm{kDa}$ ) are associated with fission (12,24). It was previously reported that leptin enhanced the accumulation of L-OPA1 via OMA1 ubiquitination, thereby inducing mitochondrial fusion, to prevent apoptosis of MSCs (9). Similarly, in the present study, significant accumulation of L-OPA1 was detected in MSCs-Lep, with higher protein expression of S-OPA1 in MSCs-Con (Fig. 2E and F), indicating that leptin promotes mitochondrial fusion through accumulation of L-OPA1.

Leptin promotes glycolysis in response to hypoxic stress. Glucose uptake and glycolysis are crucial for MSC survival, particularly in hypoxia (6). Despite the fact that leptin was found to prevent MSC apoptosis, whether glucose metabolic alterations were involved in the mechanism underlying leptin-induced MSC survival remained largely unclear. In order to determine the changes in glycolysis of MSCs, the glycogen assay was performed, which revealed that leptin induced an increase in the glycogen content of MSCs-Lep compared with MSCs-Con, as determined by PAS staining (Fig. 3A). Furthermore, cellular glucose was examined using the glucose oxidase method. In contrast to MSCs-Con, increased glucose uptake, elicited by leptin treatment, was found at the time point of hypoxic insults (Fig. 3B). Moreover, several essential genes linked to glucose metabolism were analyzed, including glyceraldehyde-3-phosphate dehydrogenase (GAPDH), hexokinase (HK)-1, lactate dehydrogenase (LDH)- $\alpha$, and pyruvate dehydrogenase (PDH)- $\alpha$, as well as key genes of fatty acid 
A
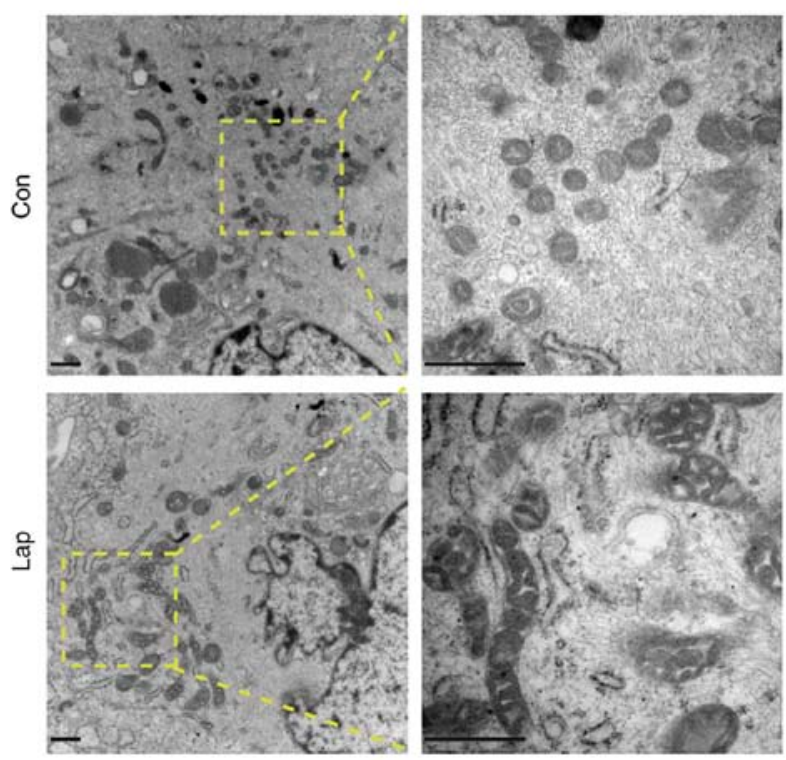

C

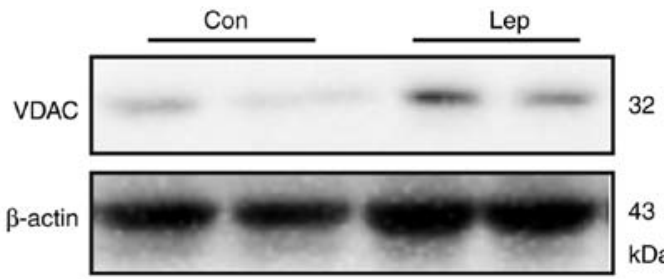

$E$

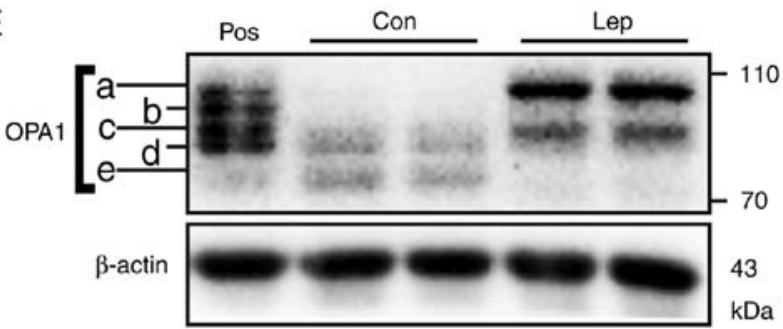

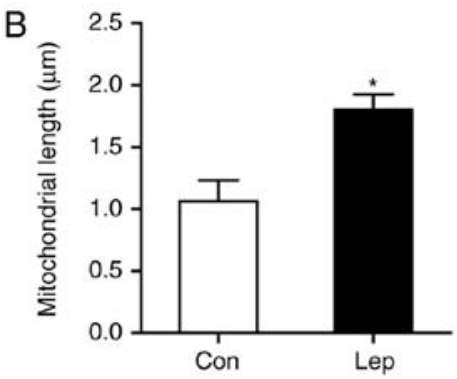

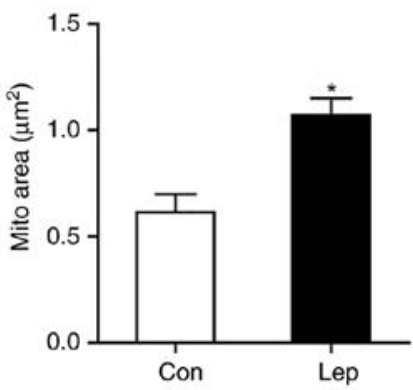

D
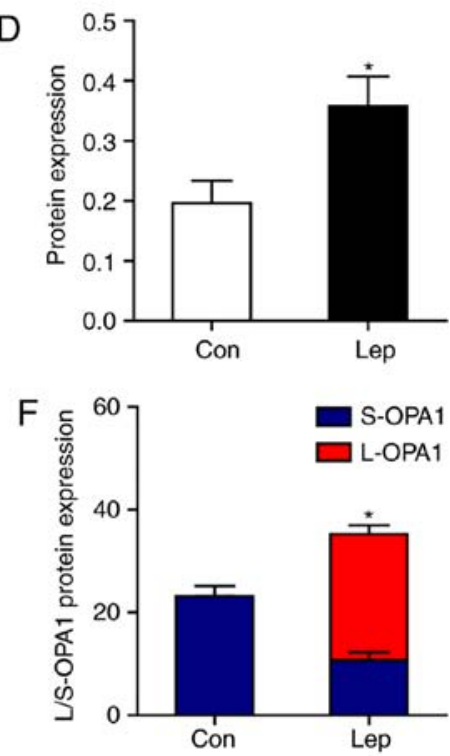

Figure 2. Enhanced mitochondrial fusion was induced by leptin treatment through L-OPA1 accumulation. (A) Representative images of mitochondrial ultrastructure were measured by TEM. Magnification was selected at x15,000 and x50,000, respectively. Scale bar: $1 \mu \mathrm{m}$. (B) Mitochondrial length and the mean mitochondrial area $\left(\mu \mathrm{m}^{2}\right)$ were calculated using Adobe Photoshop CS5 at a magnification of x30,000 (at least 20 mitochondria for each cell and at least 30 cells for each group). (C) VDAC protein expression of whole-cell lysates was detected by western blotting. Protein content was normalized using $\beta$-actin as control. (D) Densitometry was used to quantify protein expression levels. (E and F) Protein expression of different isoforms of OPA1 was evaluated by western blotting in whole-cell lysates. The expression of OPA1 in $293 \mathrm{~T}$ cells served as positive control. Different isoforms were quantified by densitometry. Each in vitro experiment was repeated three times. Data are presented as mean \pm standard error of the mean; $n=3$ ("P $<0.05)$. OPA1, optic atrophy 1 ; TEM, transmission electron microscopy; VDAC, voltage-dependent anion channel.

oxidation, such as carnitine palmitoyltransferase (cpt)-1 $\alpha(25)$ In contrast to MSCs-Con, increased gene expression of GAPDH, HK-1, LDH- $\alpha$ and PDH- $\alpha$, and decreased gene expression of cpt-1 $\alpha$, were observed in MSCs-Lep (Fig. 3C). Subsequently, the increased lactate content of the supernatant was assessed in MSCs-Lep compared with MSCs-Con (Fig. 3D) and was found to be decreased in MSCs-Lep (Fig. 3D). Furthermore, the level of adenosine triphosphate (ATP) production was higher in MSCs-Lep compared with that in MSCs-Con (Fig. 3E), and increased ECAR was measured in MSCs-Lep compared with MSCs-Con (Fig. 3F), indicating that leptin activated glycolysis to produce energy. However, a markedly lower OCR was observed in MSCs-Lep compared with MSCs-Con (Fig. 3G), demonstrating that mitochondrial OXPHOS was insufficient following leptin administration. Therefore, increased glycolysis of MSCs was observed in association with improved survival following leptin treatment under hypoxic conditions.

OPAl is involved in leptin-induced glycolysis. Improved glycolysis regulated by leptin has been confirmed based on the increased insulin sensitivity and reduction of hepatic gluconeogenesis $(8,26)$. In addition, insulin regulates glucose uptake through the Akt-mTOR-NFkB-OPA1 signaling pathway in cardiomyocytes (27). These results prompted us to investigate whether the anti-apoptotic effect of leptin was mediated via OPA1-induced glycolysis. OPA1 gene 
A Glycogen staining

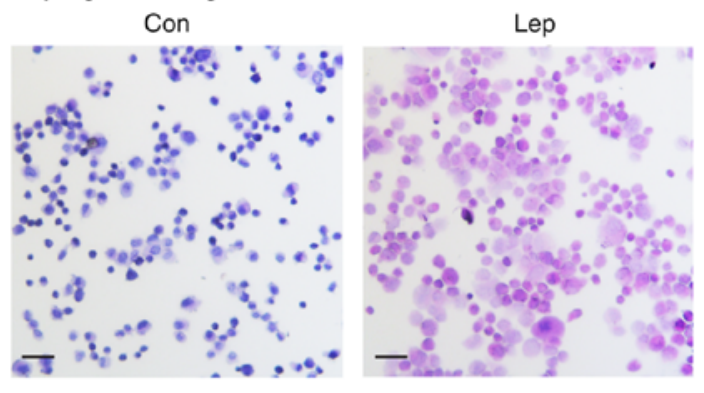

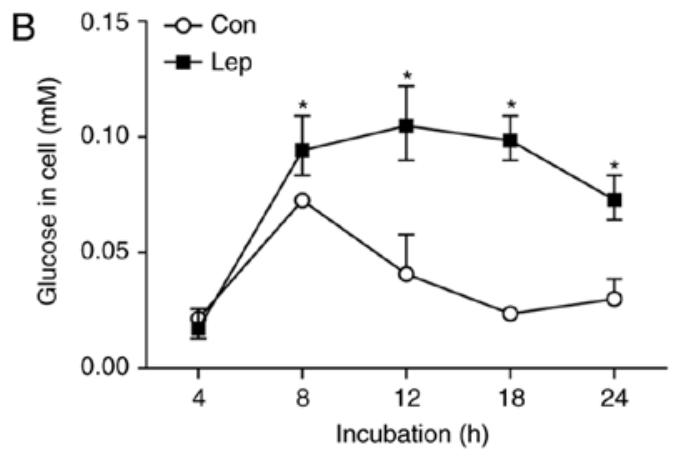

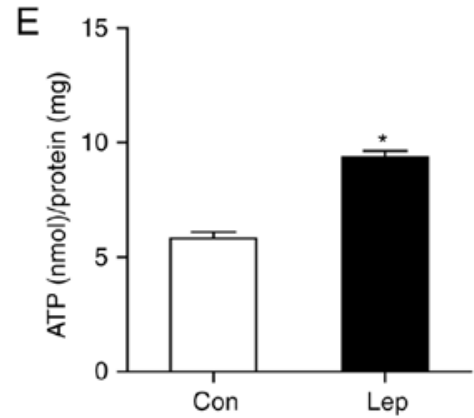

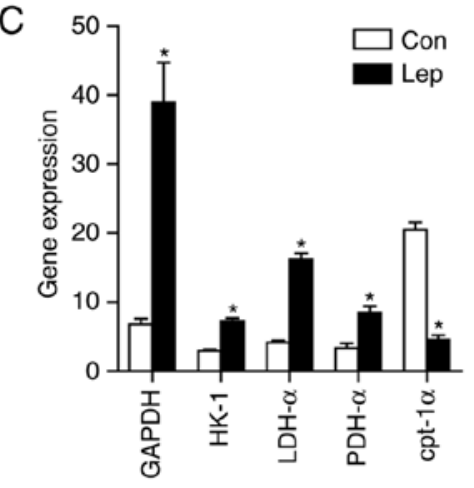

$\mathrm{F}$

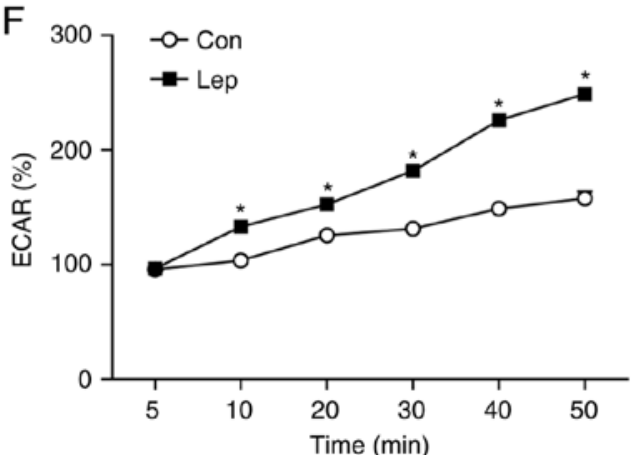

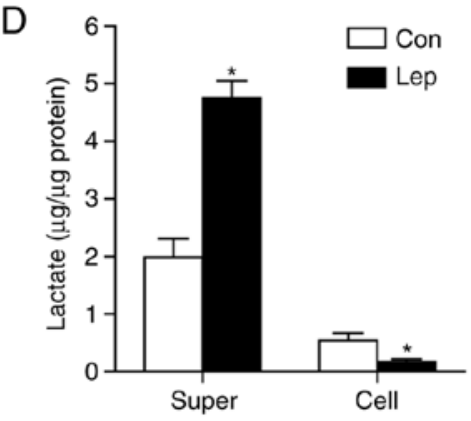

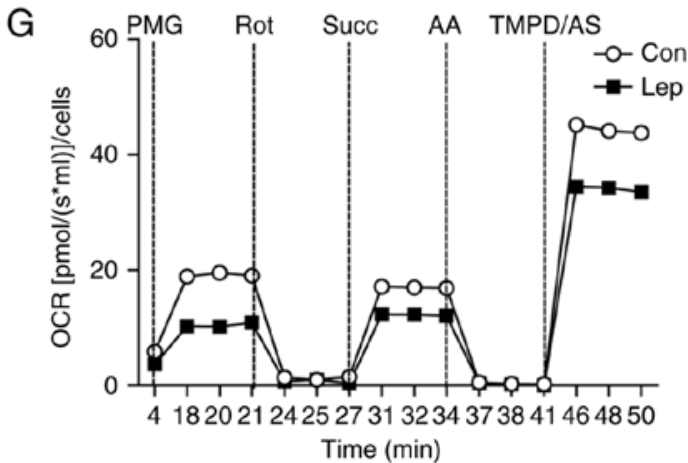

Figure 3. Glycolysis was induced by leptin treatment. (A) Following leptin treatment, MSCs were exposed to hypoxia and glucose + serum deprivation for $24 \mathrm{~h}$. Representative images of glycogen staining were revealed in MSCs-Con and MSCs-Lep by the Periodic acid-Schiff (PAS) method. Scale bar: $50 \mu$ m. (B) Glucose content was determined using the glucose oxidase method when MSCs were exposed to glucose + serum deprivation during the time course of hypoxia. (C) The gene expression of GAPDH, HK-1, LDH- $\alpha$, PDH- $\alpha$ and cpt- $1 \alpha$ was detected by RT-qPCR and normalized to $\beta$-actin. (D) Lactate content of supernatant or cellular was measured in MSCs-Con and MSCs-Lep, respectively. (E) Cellular ATP levels were measured by luciferin/luciferase-based assay, and the data were calibrated by protein content. (F) Following exposure to hypoxia and glucose + serum deprivation for 24 h, the ECAR of MSCs was measured at selected time points and is shown as percentage relative to untreated control MSCs (1x10 4 cells/96-well). (G) Mitochondrial oxygen consumption rate (OCR) was determined in MSCs-Con and MSCs-Lep using an Oroboros instrument. The data were analyzed after sequential injection of a mixture of $5 \mathrm{mM}$ pyruvate $+0.5 \mathrm{mM}$ malate $+10 \mathrm{mM}$ glutamate (PMG), $0.1 \mu \mathrm{M}$ rotenone (Rot), $10 \mathrm{mM}$ succinate (Succ), $2.5 \mu \mathrm{M}$ antimycin A (AA) and a mixture of $0.5 \mathrm{mM}$ TMPD $+2 \mathrm{mM}$ ascorbate (TMPD/AS), and calculated as the mean of each time. Data are presented as mean \pm standard error of the mean; $\mathrm{n}=3$ $($ (P<0.05). MSCs, mesenchymal stem cells; HK, hexokinase; LDH, lactate dehydrogenase; PDH, pyruvate dehydrogenase; cpt, carnitine palmitoyltransferase; RT-qPCR, reverse transcription-quantitative polymerase chain reaction; ECAR, extracellular acidification rate.

expression was silenced by small interfering RNA (siOPA1) treatment. The siOPA1 transfection efficiency was verified by western blotting (Fig. S1). In the siOPA1 group, no significant effect of leptin on glycogen staining (Fig. 4A) or the glucose content (Fig. 4B) was observed. The increase in the gene expression levels of GAPDH, HK-1, LDH- $\alpha$ and PDH- $\alpha$ following leptin treatment was blunted by OPA1 silencing, and there was no significant difference in cpt-1 $\alpha$ gene expression between the siOPA1 + Con and siOPA1 + Lep groups (Fig. 4C). Similarly, we also observed that the increased lactate levels in the supernatant and ECAR in MSCs-Lep were blunted by siOPA 1 treatment, whereas the reduced cellular lactate in MSCs-Lep started to accumulate again (Fig. 4D and F). Following siOPA1 transfection, ATP production was not altered in the siOPA 1 + Con or siOPA 1 + Lep groups, while a slight reduction in ATP was detected when comparing the $\mathrm{NC}+$ Con and siOPA $1+$ Con groups (Fig. 4E). These results indicate that improvement of leptin-induced glycolysis is primarily dependent on OPA1. Following siOPA1 transfection, the levels of OCR did not change in the siOPA1 + Con and siOPA1 + Lep groups, while they were decreased compared with the NC + Con group (Fig. 4G), which was in agreement with an earlier study reporting that OPA1 can regulate mitochondrial metabolism (28). 
A
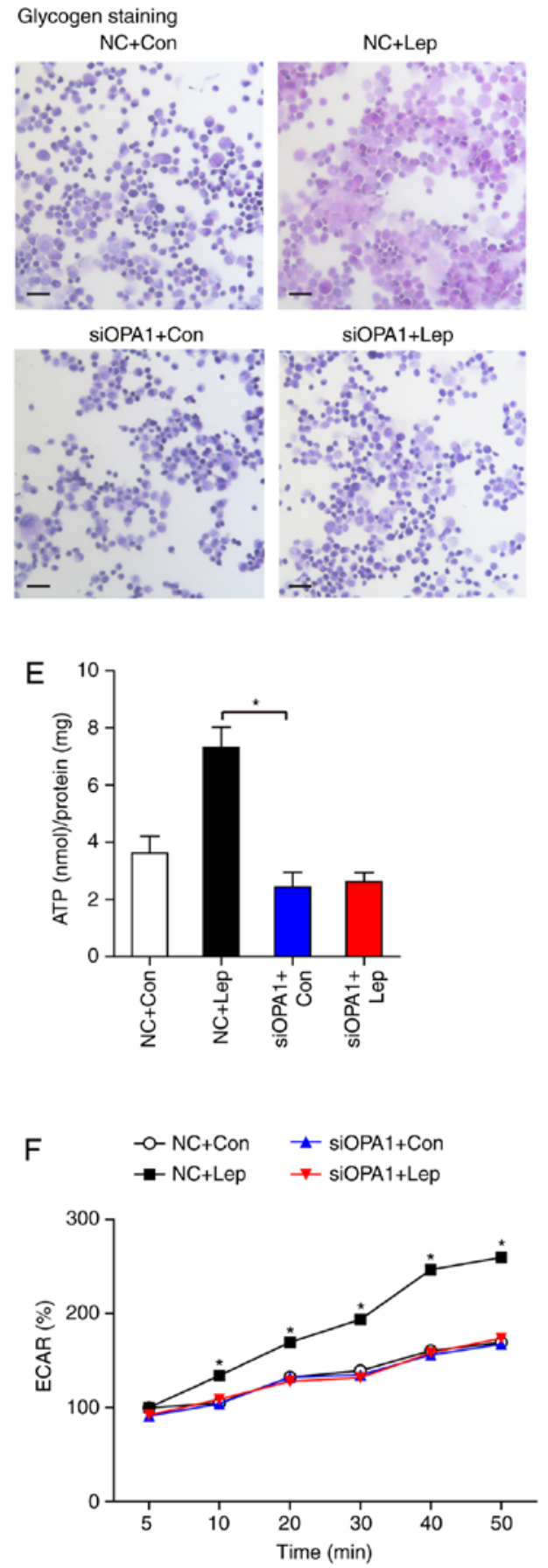

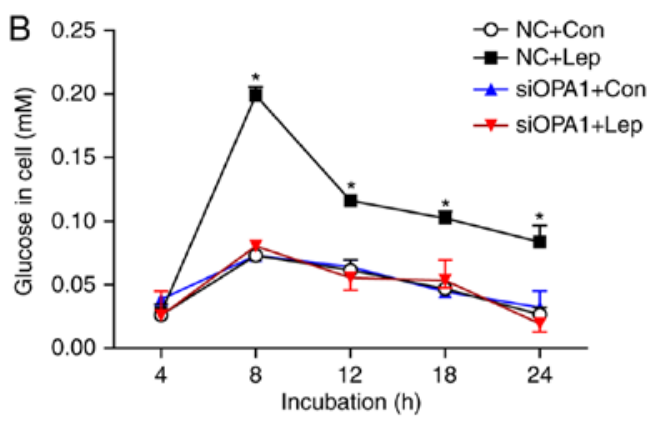

C 40 -

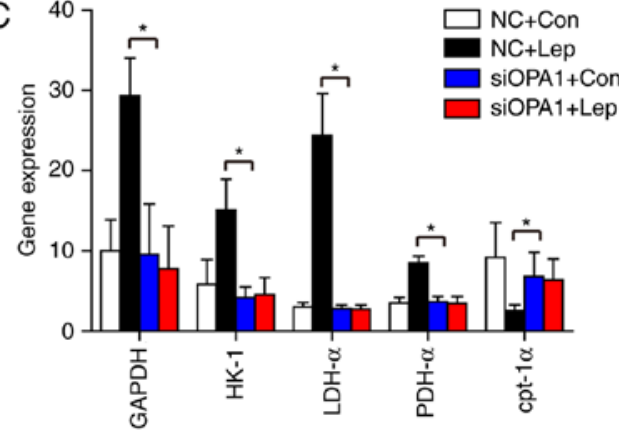

D

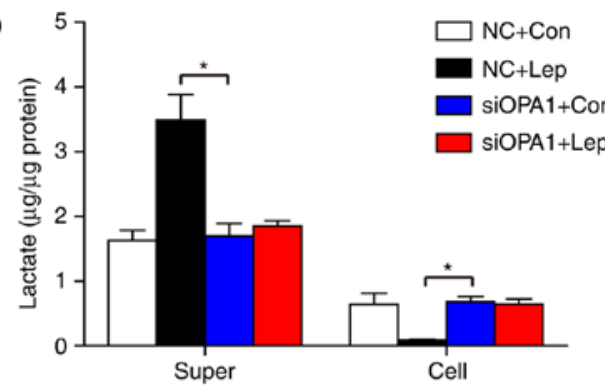

G

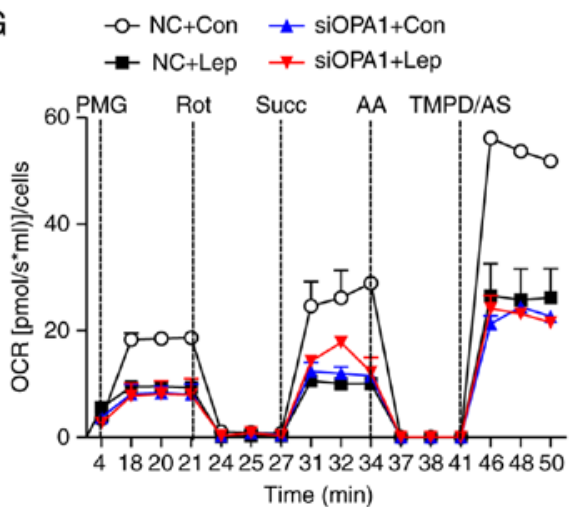

Figure 4. Leptin-induced glycolysis was required for OPA1. (A) Representative images of glycogen staining in MSCs transfected with siOPA1 in the presence or absence of leptin treatment, following exposure to hypoxia and glucose + serum deprivation for $24 \mathrm{~h}$. A scramble siRNA (NC) was used as gene silencing control. Scale bar: $50 \mu \mathrm{m}$. (B) The glucose content was analyzed in siOPA1/NC-transfected MSCs, with or without leptin treatment. (C) The mRNA level of GAPDH, HK-1, LDH- $\alpha$, PDH- $\alpha$ and cpt-1 $\alpha$ was assessed in the NC and siOPA1 groups, with or without leptin treatment, following exposure to glucose + serum deprivation during the time course of hypoxia. (D) Supernatant or cellular lactate content and (E) cellular ATP levels were measured in the NC and siOPA1 groups in the presence or absence of leptin treatment. (F) Cellular ECAR was measured at selected time points in NC + Con, NC + Lep, siOPA1 + Con and siOPA1 + Lep cells, relative to untreated control cells. (G) The OCR of the abovementioned cells was quantified using an Oroboros instrument. Each in vitro experiment was performed three times. Data are presented as mean \pm standard error of the mean; $n=3\left({ }^{*} \mathrm{P}<0.05\right)$. OPA1, optic atrophy 1 ; MSCs, mesenchymal stem cells; HK, hexokinase; LDH, lactate dehydrogenase; PDH, pyruvate dehydrogenase; cpt, carnitine palmitoyltransferase; OCR, oxygen consumption rate; ECAR, extracellular acidification rate.

Leptin increases the expression level of glucose transporters. Glucose uptake is essential for cell growth, metabolism and proliferation. Glucose diffusion across the cell membrane is accomplished by glucose transporters, including $\mathrm{Na}^{+}$-independent facilitated diffusion glucose transporters (GLUTs), sodium-glucose symporters 1 (SGLT1) and SWEETs; GLUTs and SGLT1 are responsible for glucose transport in mammals, whereas SWEETs is mainly found in plants (29). To explore how leptin regulates glucose transport, several major glucose transporters were investigated to identify the obviously increased gene expression of GLUT1, GLUT4 and SGLT1 (Fig. 5A). GLUT1 acts as 'housekeeping' 

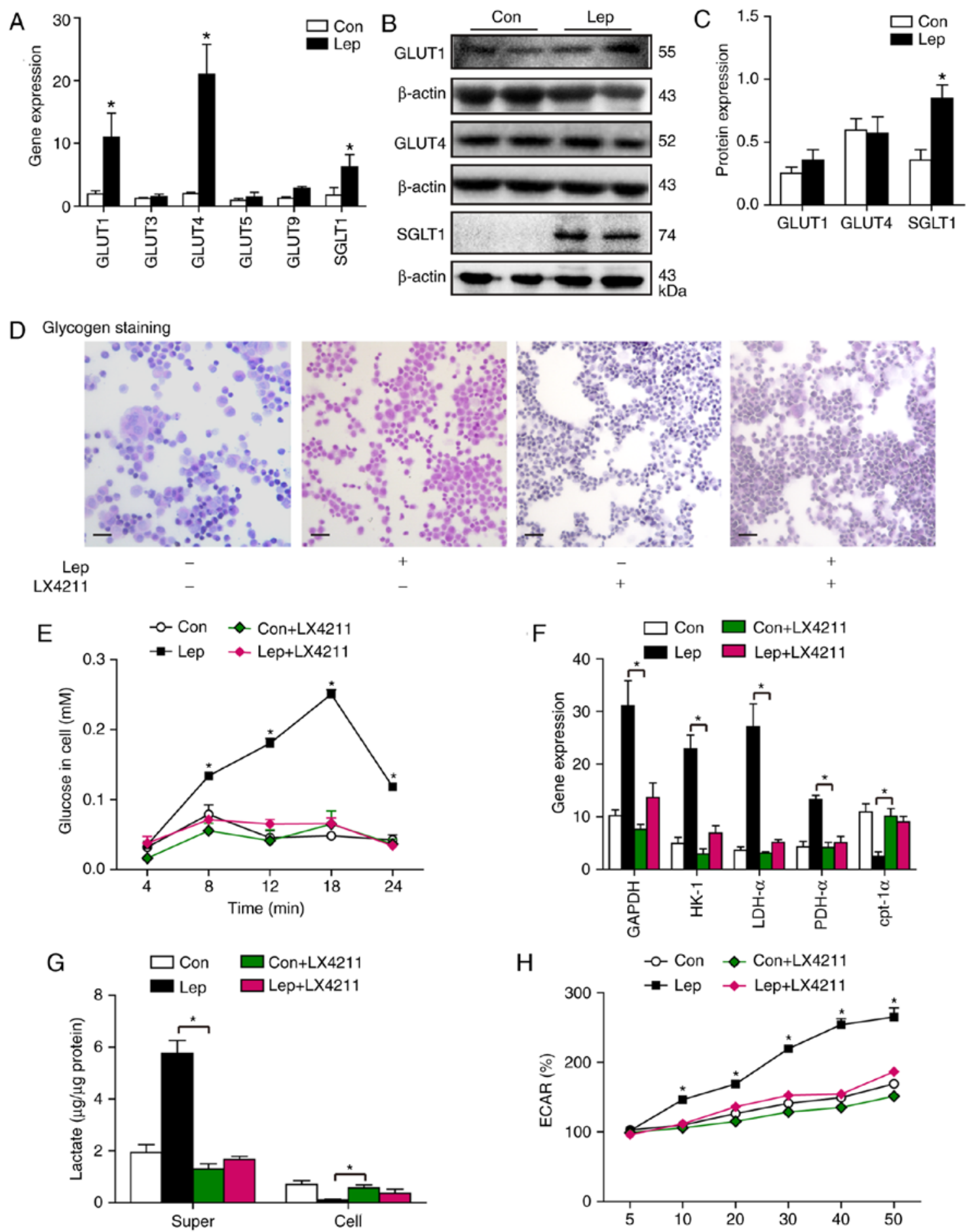

Figure 5. Leptin induced the expression of glucose transporter SGLT1. (A) Gene expression of pivotal glucose transporters, such as the GLUT family and SGLT1 after leptin administration. (B and C) The protein expression levels of GLUT1, GLUT4 and SGLT1 were assessed by western blotting and quantified using densitometry. (D and E) After incubation with leptin, the MSCs were treated with leptin in the presence or absence of $36 \mathrm{nM} \mathrm{LX4211} \mathrm{(an} \mathrm{inhibitor} \mathrm{of}$ SGLT1), with exposure to hypoxia and glucose + serum deprivation for an additional $24 \mathrm{~h}$. Glycogen staining and glucose content were evaluated in the Con, Lep, Con + LX4211 and Lep + LX4211 groups. Scale bar, $50 \mu \mathrm{m}$. (F) The mRNA expression levels of GAPDH, HK-1, LDH- $\alpha$, PDH- $\alpha$ and cpt-1 $\alpha$ under LX4211 treatment were analyzed in the abovementioned cells. $(\mathrm{G})$ The supernatant or cellular lactate content and $(\mathrm{H})$ ECAR $(\%)$ were measured in the abovementioned cells. Individual experiments were repeated three times. Data are presented as mean \pm standard error of the mean; $\mathrm{n}=3$ ("P<0.05). SGLT1, sodium-glucose symporter 1; GLUT, glucose transporters; MSCs, mesenchymal stem cells; HK, hexokinase; LDH, lactate dehydrogenase; PDH, pyruvate dehydrogenase; cpt, carnitine palmitoyltransferase; ECAR, extracellular acidification rate.

gene, as it is ubiquitous in all tissues, whereas GLUT4 is principally found in adipocytes and muscle (30). Consequently, the protein levels of GLUT1, GLUT4 and SGLT1 were measured; we observed activation of SGLT1 at the protein level in MSCs-Lep compared with MSCs-Con, while no significant impact on the expression levels of GLUT1 and GLUT4 was noted following leptin treatment (Fig. 5B and C). Subsequently, to confirm that leptin modulates glycolysis through the SGLT1 protein, leptin-treated MSCs were administered sotagliflozin (LX4211), which is as an inhibitor of SGLT1 (31). We observed 
A

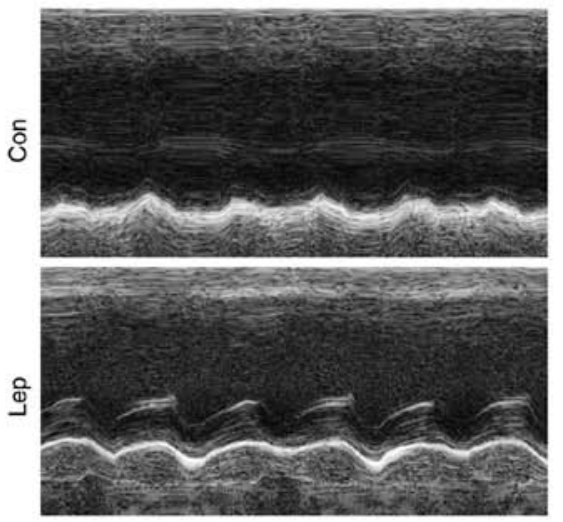

C

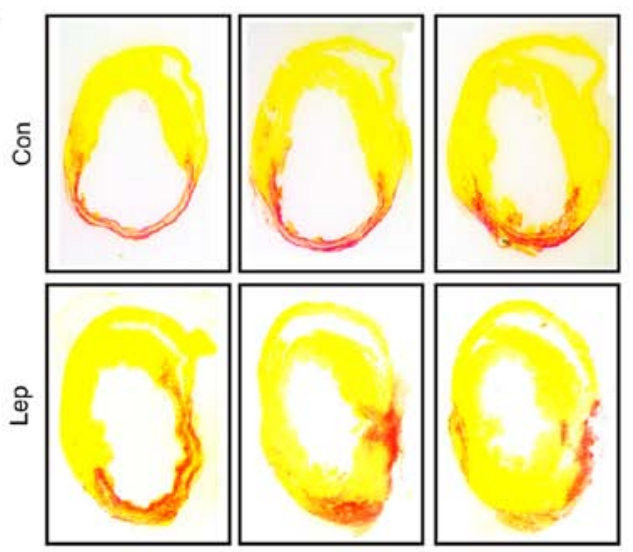

B

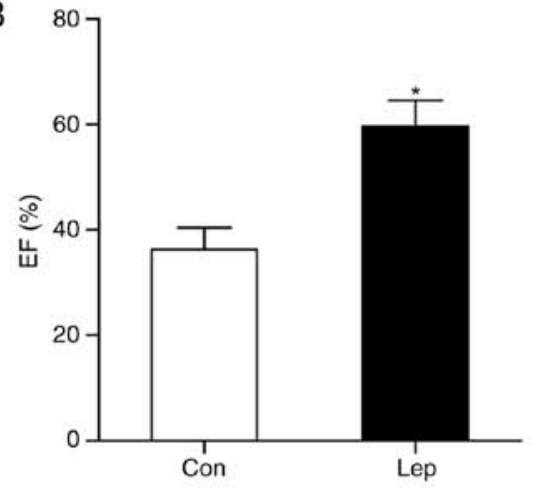

D

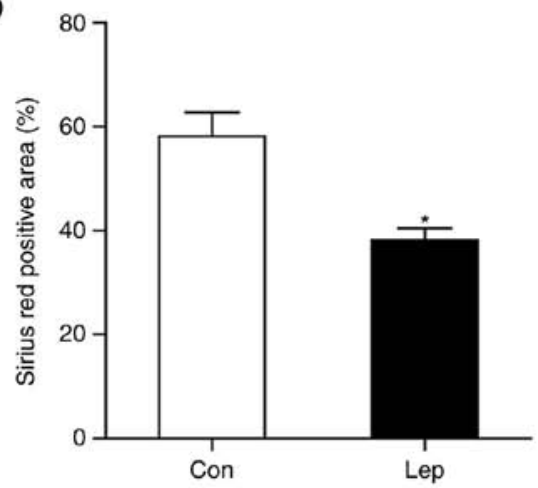

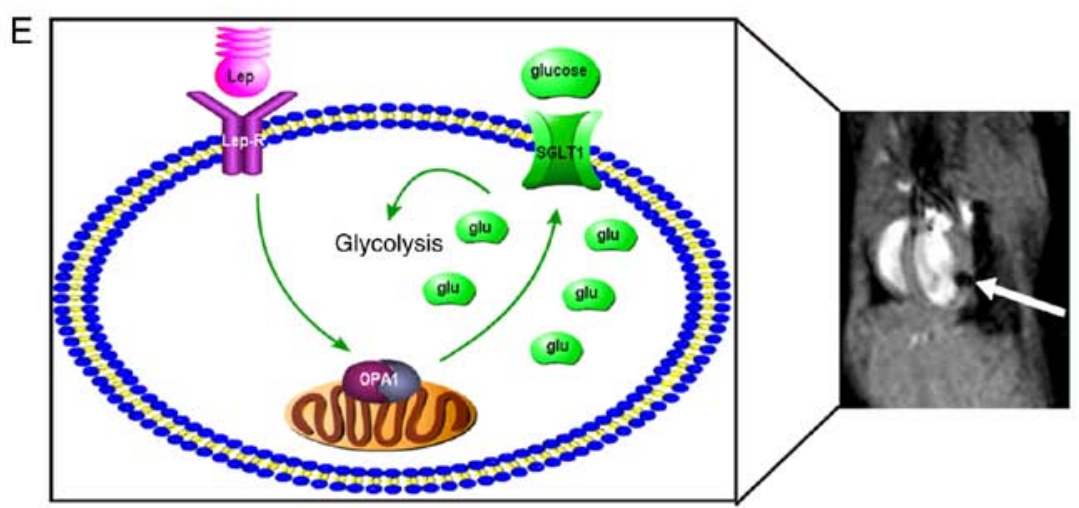

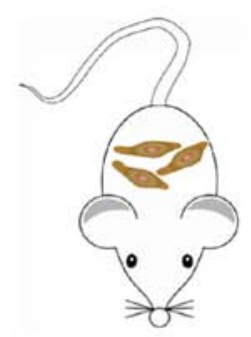

Figure 6. Transplantation of leptin-treated MSCs decreased the infarct size and rescued cardiac function following MI. (A) Echocardiography was used to assess cardiac function in an MI mouse model with transplantation of leptin-treated MSCs on day 28 post-MI. (B) Cardiac function parameters, such as EF, were measured using echocardiography ( $\mathrm{n}=5$ for the MSCs-Lep group, $\mathrm{n}=6$ for the MSCs-Con group). (C) Sirius Red staining was performed to evaluate the infarct size on day 28 post-MI. (D) The infarct size was quantification by the ratio of the area of collagen deposition over the perimeter of the left ventricle $(\mathrm{n}=5$ for the MSCs-Lep group, $\mathrm{n}=6$ for the MSCs-Con group). Data are expressed as mean \pm standard error of the mean $(" \mathrm{P}<0.05)$. (E) A schematic illustration demonstrating that leptin endowed MSCs with resistance to apoptotic death through a switch to glycolysis dependent on OPA1, primarily via activation of SGLT1. SGLT1, sodium-glucose symporter 1; MSCs, mesenchymal stem cells; MI, myocardial infarction; EF, ejection fraction.

that the increase in glucose content mediated by leptin was reversed after LX4211 administration (Fig. 5D and E). Moreover, the upregulated gene expression of GAPDH, HK-1, LDH- $\alpha$ and PDH- $\alpha$ by leptin treatment was blunted by LX4211 administration, in contrast with the unchanged gene expression of cpt-1 $\alpha$ in the Con + LX4211 and Lep + LX4211 groups (Fig. 5F). Additionally, the increased lactate content of the supernatant and the increased ECAR level mediated by leptin were blocked by LX4211 administration, while the reduction in cellular lactate accumulation following leptin treatment was hindered by LX4211 administration (Fig. 5G and H), suggesting that leptin modulates glycolysis in MSCs primarily through SGLT1 activation. However, a slightly higher lactate content of the supernatant and ECAR level were observed in the Lep + LX4211 group compared with the Con + LX4211 group (Fig. 5G and $\mathrm{H}$ ), indicating that there may be another SGLT1-independent pathway involved in leptin-mediated glycolysis, at least in part.

Survival of transplanted MSCs is improved by leptin treatment in vivo. To evaluate the leptin-mediated MSC survival in vivo, MSCs were transplanted into the border zone of the 
infarcted area in an MI mouse model. On the 28th day after MI, cardiac function was analyzed by two-dimensional echocardiography examination, and the infarct size was measured by Sirius Red staining. Notably, in contrast to MSCs-Con, improved cardiac function (Fig. 6A and B) and decreased infarct size (Fig. 6C and D) were observed in MSCs-Lep. Taken together, these data suggest that leptin endowed MSCs with apoptotic resistance through a switch to glycolysis, mediated at least in part via SGLT1, which was highly dependent on OPA1 (Fig. 6E).

\section{Discussion}

A previous study demonstrated the role of leptin in apoptotic resistance of MSCs, and demonstrated that leptin stimulated the GSK3/OMA1/OPA1 signaling pathway to promote mitochondrial fusion and protect mitochondrial integrity (9). The present study focused on a new aspect of cell metabolism. Leptin was found to improve glucose metabolism in order to provide energy for MSCs under hypoxic conditions, and the leptin-regulated glycolysis was dependent on the mitochondrial fusion protein OPA1. It was revealed that modulation of glycolysis by leptin was dependent on OPA1 primarily through activation of SGLT1. These data prompt further investigation of the association of the mitochondrial protein OPA1 with the glucose transporter SGLT1, in order to provide a pharmacological strategy of MSCs therapy for MI injury.

Additionally, accumulating evidence indicates that HPC promotes the survival of MSCs $(32,33)$. The main characteristic of the MSC microenvironment is a low-oxygen atmosphere, in which 5-10\% $\mathrm{O}_{2}$ may promote MSC proliferation (6), although differentiation of MSCs can be stimulated at $2 \% \mathrm{O}_{2}$ (34). Hypoxia-induced glycogen storage promotes the effectiveness of MSC therapy (35). Accompanied by increased lactate production rates, increased survival of MSCs may be observed in hypoxia (5). The enhanced survival of MSCs is abolished in the presence of 2DG (an inhibitor of hexokinase) (36). These findings indicate that glucose metabolism is crucial for MSC survival under hypoxic conditions. Consistent with previous studies, our results demonstrated that increased glycolysis was associated with improved MSC survival. Although long-term engraftment of transplanted MSCs has proven that only few transplanted cells survived at day 28 post-MI in a previous study (32), the therapeutic effects of MSCs are considered to be primarily due to paracrine mechanisms according to the related literature (37-39).

Leptin is a major adipostatic hormone involved in energy balance through neuroendocrine mechanisms (26). With the increasing incidence of insulin resistance and type 2 diabetes mellitus, the role of leptin in the regulation of glucose homeostasis has attracted the attention of scholars (40). Leptin improves insulin resistance and inhibits hepatic gluconeogenesis $(8,26)$. Leptin administration was shown to increase glucose infusion rate via the PI3K signaling pathway in a neuronal network (41). Moreover, leptin can increase insulin and glucagon gene expression and $\beta$-cell mass to regulate glucose homeostasis (42).

Regarding cellular energy production, mitochondria produce ATP through OXPHOS, whereas another function of mitochondria is associated with cell apoptosis (12). A sustainable mitochondrial dynamic homeostasis of fusion and fission is required for cellular health, while excessive mitochondrial fragmentation or mitophagy predispose to cell death (23). The present study demonstrated a reduction in OCR and ATP following siOPA1 treatment, with or without leptin. As a mitochondrial inner membrane protein, OPA1, plays a key role in mitochondrial respiratory efficiency and cristae remodeling (43). Additionally, in agreement with an earlier hypothesis, OPA1 regulates mitochondrial metabolism (28). In the present study, although we failed to demonstrate a restoration of mitochondrial function by leptin treatment, it may be hypothesized that the role of mitochondrial fusion in leptin-improved MSC survival was through inhibiting the release of proapoptotic proteins from the mitochondria to the cytoplasm, a phenomenon referred to as stress-induced mitochondrial hyperfusion (SIMH) (44). In addition, the decreased expression of cpt-1 $\alpha$ can inhibit fatty acids from entering the mitochondria and reduce lipogenesis, thereby stimulating glucose metabolism (45). However, cpt-1 $\alpha$ reduction may inhibit glucose oxidation without affecting glycolysis, as previously suggested (46). Taken together, these results combined with the results on the accumulation of lactate in the supernatant and the elevated levels of ECAR, indicate that the increased ATP production by leptin treatment may be primarily attributed to cellular glycolysis due to insufficient oxygen supply under hypoxic conditions. Intriguingly, a higher lactate content of the supernatant and reduced cellular lactate were detected in leptin-treated MSCs, which may be associated with MCT4 upregulation to extrude intracellular lactate and promote MSC survival in hypoxia (38).

Glucose transporters are necessary for glycolysis; however, the number of studies investigating transporter regulating networks and their activity is limited. It has also been reported that members of the GLUT family may be phosphorylated at various residues (e.g., C-terminal domain) $(29,30)$. We analyzed the mRNA levels of several major transporters, and found high expression of GLUT1, GLUT4 and SGLT1. GLUT1 acts as a 'housekeeping' gene and plays a major role in the brain, whereas GLUT4 is principally found in muscles and SGLT1 is known to be an active sodium-coupled glucose transporter in intestinal cells (30). Despite the high expression of GLUT1 and GLUT4 mRNA, higher protein levels were not detected. As previously reported (47-49), although leptin did not affect the protein levels, it activated the translocation of GLUT1 and GLUT4. Additionally, following treatment with an inhibitor of SGLT1 (LX4211), there was a mild increase in glycolysis mediated by leptin; thus, the translocation of GLUT1 and GLUT4 may be involved in leptin-modulated glycolysis. Further research on phosphorylated transporters and glucose regulatory networks is required.

In conclusion, leptin appears to play a key role in promoting MSC glycolysis in order to resist apoptosis in hypoxia. OPA1, which is associated with mitochondrial fusion, was found to be involved in leptin-modulated glycolysis, primarily through SGLT1 activation. Thus, the findings of the present study may provide a new pharmacological strategy involving leptin-regulated glucose metabolism via the OPA1 signaling pathway in MSC-based therapy for MI. 


\section{Acknowledgements}

Not applicable.

\section{Funding}

The present study was supported by the Clinical Research Center Project of the Department of Science and Technology of Guizhou Province [grant no. (2017) 5405] and the National Clinical Key Specialty Construction Project of China [grant no. (2013) 544].

\section{Availability of data and materials}

All data generated or analyzed during the present study are included in this published article.

\section{Authors' contributions}

LY: Conception and design, financial support, accountability for all aspects of the work; FY: Experiments, data analysis, figure assembly and writing of the manuscript; BL and MH: Experimental testing and data analysis; YY, XL and YZ: Manuscript revision, data analysis; HL, LZ and ST: Supervision of experimental testing; YW and LW: MI mouse model establishment; YP: Cardiac function analysis by echocardiography. All authors approved the final version of the manuscript submitted for publication.

\section{Ethics approval and consent to participate}

The experimental protocols were approved by the Human Ethics Committee of Guizhou Provincial People's Hospital.

\section{Patient consent for publication}

Not applicable.

\section{Competing interests}

The authors declare that they have no competing interests.

\section{References}

1. Lee CW, Hsiao WT and Lee OK: Mesenchymal stromal cell-based therapies reduce obesity and metabolic syndromes induced by a high-fat diet. Transl Res 182: 61-74 e8, 2017.

2. Bottcher M, Hofmann AD, Bruns H, Haibach M, Loschinski R, Saul D, Mackensen A, Le Blanc K, Jitschin R and Mougiakakos D Mesenchymal stromal cells disrupt mTOr-signaling and aerobic glycolysis during T-cell activation. Stem Cells 34: 516-521, 2016.

3. Hare JM, Traverse JH, Henry TD, Dib N, Strumpf RK, Schulman SP, Gerstenblith G, DeMaria AN, Denktas AE, Gammon RS, et al: A randomized, double-blind, placebocontrolled, doseescalation study of intravenous adult human mesenchymal stem cells (prochymal) after acute myocardial infarction. J Am Coll Cardiol 54: 2277-2286, 2009.

4. Huang XP, Sun Z, Miyagi Y, McDonald Kinkaid H, Zhang L, Weisel RD and Li RK: Differentiation of allogeneic mesenchymal stem cells induces immunogenicity and limits their long-term benefits for myocardial repair. Circulation 122: 2419-2429, 2010.

5. de Meester C, Timmermans AD, Balteau M, Ginion A Roelants V, Noppe G, Porporato PE, Sonveaux P, Viollet B, Sakamoto K, et al: Role of AMP-activated protein kinase in regulating hypoxic survival and proliferation of mesenchymal stem cells. Cardiovasc Res 101: 20-29, 2014.
6. Buravkova LB, Andreeva ER, Gogvadze V and Zhivotovsky B: Mesenchymal stem cells and hypoxia: Where are we? Mitochondrion 19: 105-112, 2014.

7. Hu X, Wu R, Jiang Z, Wang L, Chen P, Zhang L, Yang L, Wu Y, Chen $\mathrm{H}$, Chen $\mathrm{H}$, et al: Leptin signaling is required for augmented therapeutic properties of mesenchymal stem cells conferred by hypoxia preconditioning. Stem Cells 32: 2702-2713, 2014.

8. Kelesidis T, Kelesidis I, Chou S and Mantzoros CS: Narrative review: The role of leptin in human physiology: Emerging clinical applications. Ann Intern Med 152: 93-100, 2010.

9. Yang F, Wu R, Jiang Z, Chen J, Nan J, Su S, Zhang N, Wang C, Zhao J, Ni C, et al: Leptin increases mitochondrial OPA1 via GSK3-mediated OMA1 ubiquitination to enhance therapeutic effects of mesenchymal stem cell transplantation. Cell Death Dis 9: 556, 2018.

10. Pernas L and Scorrano L: Mito-morphosis: Mitochondrial fusion, fission, and cristae remodeling as key mediators of cellular function. Annu Rev Physiol 78: 505-531, 2016.

11. Head B, Griparic L, Amiri M, Gandre-Babbe S and van der Bliek AM: Inducible proteolytic inactivation of OPA1 mediated by the OMA1 protease in mammalian cells. J Cell Biol 187: 959-966, 2009.

12. Quiros PM, Langer T and Lopez-Otin C: New roles for mitochondrial proteases in health, ageing and disease. Nat Rev Mol Cell Biol 16: 345-359, 2015.

13. Wai $\mathrm{T}$ and Langer T: Mitochondrial dynamics and metabolic regulation. Trends Endocrinol Metab 27: 105-117, 2016.

14. Santoro A, Campolo M, Liu C, Sesaki H, Meli R, Liu ZW, Kim JD and Diano S: DRP1 suppresses leptin and glucose sensing of POMC neurons. Cell Metab 25: 647-660, 2017.

15. Buck MD, O'Sullivan D, Klein Geltink RI, Curtis JD, Chang CH, Sanin DE, Qiu J, Kretz O, Braas D, van der Windt GJ, et al: Mitochondrial dynamics controls T cell fate through metabolic programming. Cell 166: 63-76, 2016

16. Nan J, Hu H, Sun Y, Zhu L, Wang Y, Zhong Z, Zhao J, Zhang N, Ye J, Wang Y, et al: TNFR2 stimulation promotes mitochondrial fusion via stat3- and NF-kB-dependent activation of OPA1 expression. Circ Res 121: 392-410, 2017.

17. Kuznetsov AV, Veksler V, Gellerich FN, Saks V, Margreiter R and Kunz WS: Analysis of mitochondrial function in situ in permeabilized muscle fibers, tissues and cells. Nat Protoc 3: 965-976, 2008.

18. Zhang N, Ye F, Zhu W, Hu D, Xiao C, Nan J, Su S, Wang Y, Liu M, Gao K, et al: Cardiac ankyrin repeat protein attenuates cardiomyocyte apoptosis by upregulation of $\mathrm{Bcl}-2$ expression. Biochim Biophys Acta 1863: 3040-3049, 2016.

19. Wang C, Chen H, Zhu W, Xu Y, Liu M, Zhu L, Yang F, Zhang L, Liu X, Zhong Z, et al: Nicotine accelerates atherosclerosis in apolipoprotein E-deficient mice by activating $\alpha 7$ nicotinic acetylcholine receptor on mast cells. Arterioscler Thromb Vasc Biol 37: 53-65, 2017.

20. Liu X, Hu D, Zeng Z, Zhu W, Zhang N, Yu H, Chen H, Wang K, Wang Y, Wang L, et al: SRT1720 promotes survival of aged human mesenchymal stem cells via FAIM: A pharmacological strategy to improve stem cell-based therapy for rat myocardial infarction. Cell Death Dis 8: e2731, 2017.

21. Lahera V, de Las Heras N, Lopez-Farre A, Manucha W and Ferder L: Role of mitochondrial dysfunction in hypertension and obesity. Curr Hypertens Rep 19: 11, 2017.

22. Giuliano M, Lauricella M, Calvaruso G, Carabillò M, Emanuele S, Vento R and Tesoriere G: The apoptotic effects and synergistic interaction of sodium butyrate and MG132 in human retinoblastoma Y79 cells. Cancer Res 59: 5586-5595, 1999.

23. Chan DC: Fusion and fission: Interlinked processes critical for mitochondrial health. Annu Rev Genet 46: 265-287, 2012.

24. Quirós PM, Ramsay AJ, Sala D, Fernández-Vizarra E, Rodríguez F, Peinado JR, Fernández-García MS, Vega JA, Enríquez JA, Zorzano A and López-Otín C: Loss of mitochondrial protease OMA1 alters processing of the GTPase OPA1 and causes obesity and defective thermogenesis in mice. EMBO J 31: 2117-2133, 2012.

25. Li Y, Wong K, Giles A, Jiang J, Lee JW, Adams AC, Kharitonenkov A, Yang Q, Gao B, Guarente L and Zang M: Hepatic SIRT1 attenuates hepatic steatosis and controls energy balance in mice by inducing fibroblast growth factor 21 . Gastroenterology 146: 539-549e7, 2014.

26. Flier JS, Harris M and Hollenberg AN: Leptin, nutrition, and the thyroid: The why, the wherefore, and the wiring. J Clin Invest 105: 859-861, 2000. 
27. Parra V, Verdejo HE, Iglewski M, Del Campo A, Troncoso R, Jones D, Zhu Y, Kuzmicic J, Pennanen C, Lopez-Crisosto C, et al: Insulin stimulates mitochondrial fusion and function in cardiomyocytes via the Akt-mTOR-NFкB-Opa-1 signaling pathway. Diabetes 63: 75-88, 2014.

28. Patten DA, Wong J, Khacho M, Soubannier V, Mailloux RJ, Pilon-Larose K, MacLaurin JG, Park DS, McBride HM, Trinkle-Mulcahy L, et al: OPA1-dependent cristae modulation is essential for cellular adaptation to metabolic demand. EMBO J 33: 2676-2691, 2014

29. Chen LQ, Cheung LS, Feng L, Tanner W and Frommer WB: Transport of sugars. Annu Rev Biochem 84: 865-894, 2015.

30. Deng D, Sun P, Yan C, Ke M, Jiang X, Xiong L, Ren W, Hirata K, Yamamoto M, Fan S and Yan N: Molecular basis of ligand recognition and transport by glucose transporters. Nature 526: 391-396, 2015

31. Zambrowicz B, Freiman J, Brown PM, Frazier KS, Turnage A, Bronner J, Ruff D, Shadoan M, Banks P, Mseeh F, et al: LX4211, a dual SGLT1/SGLT2 inhibitor, improved glycemic control in patients with type 2 diabetes in a randomized, placebo-controlled trial. Clin Pharmacol Ther 92: 158-169, 2012.

32. Hu X, Xu Y, Zhong Z, Wu Y, Zhao J, Wang Y, Cheng $\mathrm{H}$, Kong M, Zhang F, Chen Q, et al: A large-scale investigation of hypoxia-preconditioned allogeneic mesenchymal stem cells for myocardial repair in nonhuman primates: Paracrine activity without remuscularization. Circ Res 118: 970-983, 2016.

33. Lavrentieva A, Majore I, Kasper C and Hass R: Effects of hypoxic culture conditions on umbilical cord-derived human mesenchymal stem cells. Cell Commun Signal 8: 18, 2010.

34. Volkmer E, Kallukalam BC, Maertz J, Otto S, Drosse I, Polzer H, Bocker W, Stengele M, Docheva D, Mutschler W and Schieker M: Hypoxic preconditioning of human mesenchymal stem cells overcomes hypoxia-induced inhibition of osteogenic differentiation. Tissue Eng Part A 16: 153-164, 2010.

35. Zhu H, Sun A, Zou Y and Ge J: Inducible metabolic adaptation promotes mesenchymal stem cell therapy for ischemia: A hypoxia-induced and glycogen-based energy prestorage strategy. Arterioscler Thromb Vasc Biol 34: 870-876, 2014

36. Mylotte LA, Duffy AM, Murphy M, O'Brien T, Samali A, Barry F and Szegezdi E: Metabolic flexibility permits mesenchymal stem cell survival in an ischemic environment. Stem Cells 26: 1325-1336, 2008.

37. Hsiao ST, Asgari A, Lokmic Z, Sinclair R, Dusting GJ, Lim SY and Dilley RJ: Comparative analysis of paracrine factor expression in human adult mesenchymal stem cells derived from bone marrow, adipose, and dermal tissue. Stem Cells Dev 21: 2189-2203, 2012.
38. Saraswati S, Guo Y, Atkinson J and Young PP: Prolonged hypoxia induces monocarboxylate transporter-4 expression in mesenchymal stem cells resulting in a secretome that is deleterious to cardiovascular repair. Stem Cells 33: 1333-1344, 2015.

39. Tachibana A, Santoso MR, Mahmoudi M, Shukla P, Wang L, Bennett M, Goldstone AB, Wang M, Fukushi M, Ebert AD, et al: Paracrine effects of the pluripotent stem cell-derived cardiac myocytes salvage the injured myocardium. Circ Res 121: e22-e36, 2017.

40. Morton GJ and Schwartz MW: Leptin and the central nervous system control of glucose metabolism. Physiol Rev 91: 389-411, 2011.

41. Rasmussen BA, Breen DM, Duca FA, Côté CD, ZadehTahmasebi M, Filippi BM and Lam TK: Jejunal leptin-PI3K signaling lowers glucose production. Cell Metab 19: 155-161, 2014.

42. Michel M, Page-McCaw PS, Chen W and Cone RD: Leptin signaling regulates glucose homeostasis, but not adipostasis, in the zebrafish. Proc Natl Acad Sci USA 113: 3084-3089, 2016.

43. Varanita T, Soriano ME, Romanello V, Zaglia T, QuintanaCabrera R, Semenzato M, Menabò R, Costa V, Civiletto G, Pesce $\mathrm{P}$, et al: The opa1-dependent mitochondrial cristae remodeling pathway controls atrophic, apoptotic, and ischemic tissue damage. Cell Metab 21: 834-844, 2015.

44. Tondera D, Grandemange S, Jourdain A, Karbowski M, Mattenberger Y, Herzig S, Da Cruz S, Clerc P, Raschke I, Merkwirth C, et al: SLP-2 is required for stress-induced mitochondrial hyperfusion. EMBO J 28: 1589-1600, 2009.

45. Bertero E and Maack C: Metabolic remodelling in heart failure. Nat Rev Cardiol 15: 457-470, 2018.

46. Gamble J and Lopaschuk GD: Glycolysis and glucose oxidation during reperfusion of ischemic hearts from diabetic rats. Biochim Biophys Acta 1225: 191-199, 1994.

47. Benomar Y, Naour N, Aubourg A, Bailleux V, Gertler A, Djiane J, Guerre-Millo M and Taouis M: Insulin and leptin induce Glut4 plasma membrane translocation and glucose uptake in a human neuronal cell line by a phosphatidylinositol 3-kinase-dependent mechanism. Endocrinology 147: 2550-2556, 2006.

48. Berti L, Kellerer M, Capp E and Häring HU: Leptin stimulates glucose transport and glycogen synthesis in $\mathrm{C} 2 \mathrm{C} 12$ myotubes: Evidence for a PI3-kinase mediated effect. Diabetologia 40: 606-609, 1997.

49. Berti L and Gammeltoft S: Leptin stimulates glucose uptake in $\mathrm{C} 2 \mathrm{C} 12$ muscle cells by activation of ERK2. Mol Cell Endocrinol 157: 121-130, 1999. 